\title{
Balancing ACT: evaluating the effectiveness of psychoeducation and Acceptance and Commitment Therapy (ACT) groups for people with bipolar disorder: study protocol for pilot randomised controlled trial
}

Emma O'Donoghue ${ }^{1,5^{*}} \mathbb{D}$, Abigail Clark ${ }^{1}$, Matthew Richardson ${ }^{1}$, John Hodsoll ${ }^{2}$, Sunil Nandha ${ }^{1}$, Eric Morris ${ }^{3}$, Fergus Kane ${ }^{4}$, Deirdre O'Keeffe ${ }^{1}$, Lucy Butler ${ }^{1}$ and Suzanne Jolley ${ }^{1,4}$

\begin{abstract}
Background: Bipolar disorder is a chronic and disabling psychiatric condition, characterised by recurrent episodes of mania, hypomania and depression. It places a heavy burden on sufferers and families, with high societal and healthcare costs. Many service users with a diagnosis of bipolar disorder also experience prominent psychotic symptoms, with differential diagnoses of schizoaffective disorder, and relapses characterised by repeated manic psychotic episodes and grandiosity. Such presentations require specific adaptations to standard bipolar disorder interventions in order to address their psychosis, alongside mood regulation, with a particular emphasis on impulsivity, irritability, disinhibition and elation. The Balancing ACT study aims to evaluate an innovative group intervention combining Acceptance and Commitment Therapy and psychoeducation approaches (ACT/PE) with individuals experiencing bipolar disorder and/or symptoms within community psychosis services.
\end{abstract}

Methods: The Balancing ACT study is a randomised controlled trial comparing Balancing ACT groups (ACT/PE) plus routine care to routine care alone. Balancing ACT (ACT/PE) comprises ten group sessions, each lasting 2 hours, delivered weekly. The primary outcome is psychological wellbeing; secondary outcomes are mental health relapses (measured by service use averages for the 12 months pre baseline and 3 months post baseline). We will also measure mood, distress, recovery and psychological change processes. Participants will be randomised in a 1:1 ratio, after baseline assessment. Outcomes will be assessed by trained assessors blind to treatment condition at 0 , 10 and 14 weeks. Recruitment began in April 2017 and is on-going until the end of October 2017.

Discussion: The Balancing ACT study will contribute to the currently limited evidence base for psychological interventions for people experiencing bipolar disorder and/or symptoms in the context of community psychosis services.

Trial registration: ISRCTN73327972. Registered on 27 March 2017. Balancing ACT: evaluating the effectiveness of psychoeducation and Acceptance and Commitment Therapy (ACT) groups for people with bipolar disorder.

Keywords: Bipolar disorder, Community mental health, Acceptance and Commitment Therapy (ACT), Psychosis, Psychoeducation

\footnotetext{
* Correspondence: Emma.ODonoghue@slam.nhs.uk

${ }^{1}$ South London and Maudsley NHS Foundation Trust, London SE5 8AZ, UK

${ }^{5}$ South London and Maudsley NHS Foundation Trust, 308-312 Brixton Road, London SW9 6AA, UK

Full list of author information is available at the end of the article
}

(C) The Author(s). 2018 Open Access This article is distributed under the terms of the Creative Commons Attribution 4.0 International License (http://creativecommons.org/licenses/by/4.0/), which permits unrestricted use, distribution, and reproduction in any medium, provided you give appropriate credit to the original author(s) and the source, provide a link to the Creative Commons license, and indicate if changes were made. The Creative Commons Public Domain Dedication waiver (http://creativecommons.org/publicdomain/zero/1.0/) applies to the data made available in this article, unless otherwise stated. 


\section{Background}

Bipolar disorder is a chronic and disabling psychiatric condition, characterised by recurrent episodes of mania, hypomania and depression. It places a heavy burden on sufferers and families, and has high societal and healthcare costs [1,2]. An estimated 2 to $6 \%$ of the general population in the UK meet the criteria for bipolar disorder, costing $£ 5.2$ billion annually in healthcare and lost employment [3]. Many of the service users with a diagnosis of bipolar disorder also experience prominent psychotic symptoms, with differential diagnoses of schizoaffective disorder, and relapses characterised by repeated manic psychotic episodes and grandiosity. Such presentations require specific adaptations to standard psychological interventions for bipolar disorder in order to address psychosis, alongside mood regulation, with a particular emphasis on impulsivity, irritability, disinhibition and elation [2].

The main treatment offered for bipolar disorder is medication [2]. Despite this, many individuals continue to experience residual symptoms, even when they are fully concordant with medication, with rates of relapse of between 40 and $60 \%[2,4,5]$. Psychological therapies were previously thought to add little to the treatment of severe mental illness. However, psychological interventions for people with psychosis are now firmly established as clinical and cost-effective interventions, and, although the move away from a predominantly medical approach has been more recent for bipolar disorder, there is increasing evidence that patients benefit from high-quality psychological therapies, with clinical and cost effects, as well as improving the quality of life of service users and their families [6]. The National Institute for Health and Care Excellence [2] guidelines recommend up to 16 sessions of individual cognitive behaviour therapy (CBT) including psychoeducation and self-management/relapse prevention for bipolar disorder and the same duration of intervention for people with psychosis, incorporating self-monitoring, coping strategies, distress-reduction and improving functioning. Family Interventions (FI) and psychosocial/vocational support are also recommended for both bipolar disorder and psychosis.

A principal goal of psychoeducation for bipolar disorder is to provide accurate and reliable information, as well as teaching early recognition and self-management skills and facilitating service users to make informed decisions about the management of their own mental healthcare within the context of a collaborative working relationship with their clinical team [7]. Psychoeducation is usually facilitated in a group format and has also been found to be helpful for family members and caregivers of people with bipolar disorder, by reducing relapses and increasing time spent well $[7,8]$.
Psychoeducation for service users with bipolar disorder with mania and psychosis symptoms requires accurate and detailed information about psychosis, recovery, and a particular focus on symptoms of elevated mood and grandiosity. Traditional approaches to psychoeducation (e.g. teaching people about the medical nature of their condition) alone for people with psychosis have been found to be ineffective [9]. This could possibly be due to the difficulty of establishing a shared understanding between the service user and the clinical team in the context of differing levels of insight. Psychoeducation for this group must accommodate these specific engagement issues to develop individually tailored, validating and normalising understandings that do not inadvertently discredit personally valued unusual experiences and beliefs. Psychoeducational material of this kind has yet to be formally evaluated.

Acceptance and Commitment Therapy (ACT) is an emerging psychological intervention which facilitates self-management and acceptance of difficulties, and promotes a focus on valued personal goals, in the face of adversity [10]. ACT also promotes social inclusion by shifting the service user's focus from symptom control to connecting with personal values and participating in life. The central tenets of ACT, and its empowering, equitable, open and collaborative therapeutic stance, therefore fit it well to a recovery ethos. Unlike many psychological interventions, ACT relies on a philosophy which can be readily trained, and a recent pilot study by our group in South London and Maudsley NHS Foundation Trust, funded by the Maudsley Charity, demonstrated that co-facilitators could learn to deliver ACT by co-facilitating groups over a 4-week course of therapy (Jolley S, Johns L, O’Donoghue E, Oliver J, Khondoker M, Byrne M, Butler L, De Rosa C, Sim F, Morris EM: A randomised controlled trial of group acceptance and commitment therapy for patients and caregivers in psychosis services, Submitted).

ACT has shown good preliminary evidence of effectiveness for people with psychosis $[11,12]$ and ACT trials have contributed to the recommendations of the NICE guidance [13]. ACT has recently been piloted for people with bipolar disorder who experience co-existing anxiety and found to be effective [14]. However, group ACT and psychoeducation interventions for people with bipolar disorder have not yet been evaluated in a randomised controlled trial (RCT). ACT's transdiagnostic nature fits well with mood instability alongside psychotic symptoms: $\mathrm{ACT}$ aims to target transdiagnostic processes, and recent studies indicate the potential effectiveness of ACT across a broad spectrum of severe mental health conditions, including mood instability and psychotic symptoms $[11,12]$. ACT frames values, in particular, as remaining relatively constant throughout a person's life: mood episodes and other problematic symptoms lead to the person losing touch with their values, rather than fundamentally 
changing them. During depressive, hypomanic and manic episodes, it is common for people to engage in activities that are contrary to their normal values and/or to drop activities that are in line with such values [15]. Such changes may be driven, maintained and amplified by cognitive distortions, including mood-consistent altered perceptions of the future $[15,16]$, as well as experiential avoidance of painful emotions, and reduced meta-cognitive awareness. An explicit focus on values aims to help people stay in touch with said values during prodromal mood states, and thus remain engaged with 'value-driven action', helping prevent escalation into mania or descent into depression.

The values component of ACT helps people to get in touch with chosen life directions, which will be present regardless of an individual's mood state; values provide a source of reinforcement and goal-directed activity independent of mood, as well as having elements that are recovery-consistent. Accordingly, recent studies indicate the potential for effectiveness across a broad spectrum of severe mental health conditions [11, 12].

\section{Study aims}

We plan to carry out a pilot RCT to test out the potential feasibility and clinical and cost-effectiveness of our Balancing ACT groups incorporating ACT and psychoeducation (ACT/PE), as an adjunct to routine care (treatment as usual, TAU), in improving psychological wellbeing in adults (aged 18 years or over) with a diagnosis of bipolar disorder and/or bipolar symptoms in community psychosis services. The Balancing ACT (ACT/PE) intervention plus TAU condition will be compared to TAU alone. Service users allocated to TAU will be offered the Balancing ACT intervention after completing the final study assessment.

The specific research questions to be addressed are:

1) Are primary and secondary clinical outcomes for people with bipolar disorder and/or bipolar symptoms in psychosis community services potentially improved by the addition of ACT/PE groups? If so, to what extent, and with what likely variability in effect size?

2) Do the ACT/PE groups have a potential positive impact on mood, distress, recovery and psychological processes of change?

3) Is the ACT/PE intervention potentially cost-effective?

4) Is it feasible to add ACT/PE approaches to bipolar disorder psychological treatments?

\section{Methods}

\section{Participants and setting}

We aim to recruit 36 adults aged 18 years or over, presenting to the Promoting Recovery Service Community
Mental Health Teams of the Psychosis Clinical Academic Group of the South London and Maudsley NHS Foundation Trust (SLaM) within King's Health Partners. Ten teams, from the inner-London boroughs of Lambeth and Southwark are participating. The sample will include service users with diagnoses of bipolar affective disorder and schizoaffective disorder. A recent local audit showed that of 1344 adults currently treated in SLaM with a diagnosis of bipolar disorder, more than half $(N=823)$ are being treated within psychosis services, with around 200 in each borough (13-20\% of the caseload). As such, we expect recruitment to be reasonably straightforward.

The proposed sample size, while representing only a small proportion of the caseload, is in line with recommendations for the number of participants required to estimate variance in outcomes and is, therefore, appropriate for an early stage feasibility study. (24 to 50 participants $[17,18])$. The feasibility study is designed to feed into a second larger pilot study, which will benefit from feedback received in the current study, and allow consideration of the representativeness of the study sample.

\section{Inclusion and exclusion criteria}

Inclusion criteria are: presenting to, and being treated under, Lambeth or Southwark Promoting Recovery Services (the services see working age adults, aged 18 years or over); diagnosis of bipolar disorder and/or bipolar symptoms; being available for the study duration; sufficient English language ability to be able to complete assessment measures and participate in therapy, without interpreter support (as this cannot feasibly be provided in a group context). Exclusion criteria are: inability to remain in a group setting, attend and understand and interact for up to $2 \mathrm{~h}$; considered by the treating team to lack capacity to consent. This will mean that people with acute extremes of mood state are unlikely to meet the inclusion criteria, although an extreme mood state will not be an exclusion criterion in itself.

\section{Study design}

Balancing ACT is an RCT with random allocation to one of two arms, comparing our active intervention (ACT/PE + TAU) to routine care alone (TAU). TAU will be delivered without interference in both conditions and includes care coordination, medication, and practical/ emotional support for the individual. We will record what is delivered as TAU. Assessments will take place at baseline ( 0 weeks), 10 weeks (post therapy) and 14 weeks (post-booster session). After 14 weeks, TAU participants will be offered the intervention. Trained research workers will complete assessments with participants. Baseline assessments will be carried out prior to randomisation; 10- and 14-week assessments will be arranged by the trial 
research worker, but carried out by a different assessor, who will be blind to treatment allocation. Service measures [19] will be completed for the 12-month period before baseline and 3 months after the final baseline assessment. The study design is illustrated in Fig. 1.

\section{Procedure}

\section{Recruitment}

We will recruit directly from Promoting Recovery Services in Lambeth and Southwark boroughs. Adults presenting with bipolar disorder and/or symptoms will be invited by their team to find out more about the study, and, if agreeing, will be contacted by the Balancing ACT study researcher. Telephone conversations and meetings will take place as needed to discuss the study. Participants will be provided with an information sheet and consent form. If an individual consents to participate, they will be offered a baseline assessment and will be randomised only following completion of this.

\section{Ethical approval}

The study has been reviewed and given a favourable opinion by the London-Surrey Borders National Research Ethics Committee (REC Reference: 17/LO/04/45).

\section{Intervention}

The ACT/PE intervention will consist of ten groups, each running over ten consecutive weeks, followed by a booster session, 4 weeks later. Each group session will last for $2 \mathrm{~h}$. The intervention will be delivered in addition to routine care (specialist care coordination, medication, practical and emotional support), and will be compared to routine care alone (TAU). After the 14-week assessment, individuals in the routine care condition will be offered the intervention.

The ACT/PE intervention will focus on psychoeducation approaches to understanding bipolar and mood disorders. Each session will focus on a different issue pertinent to bipolar disorder; including, mania, stress, sleep, depression, medication, communicating with friends and family, and relapse prevention. Each session will introduce ACT skills as a different way of responding to symptoms of bipolar disorder. The Balancing ACT groups are designed to be interactive with experiential exercises throughout and a commitment to work towards a valued goal, and the practice mindfulness exercises in between sessions.

We acknowledge that 14 weeks is a relatively short timeframe to assess change. For the pragmatic purposes of this study, because we are interested in the potential helpfulness of the intervention, rather than the longevity of the effects, we consider this time period appropriate.

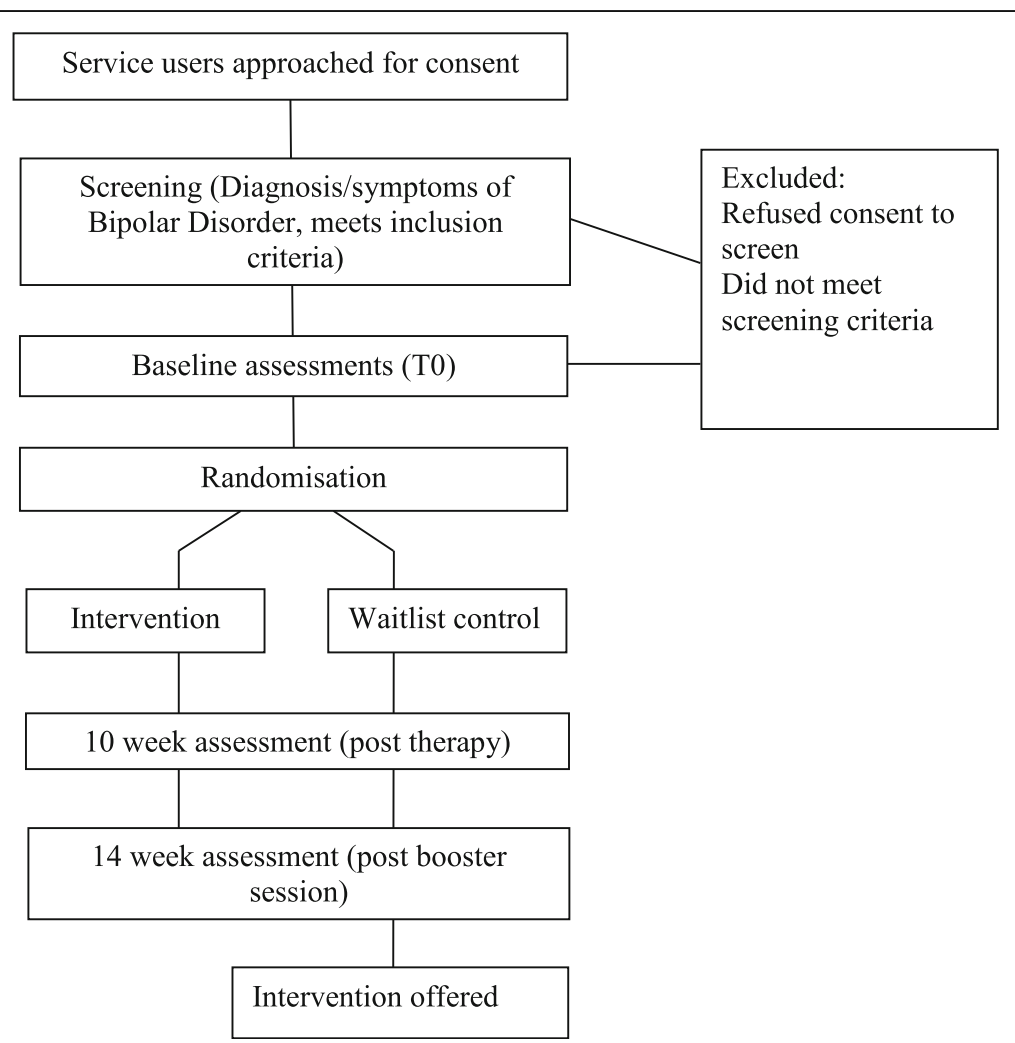

Fig. 1 Balancing ACT: evaluating the effectiveness of psychoeducation and Acceptance and Commitment Therapy (ACT) groups for people with bipolar disorder: study protocol for a pilot randomised controlled trial. Study design 
ACT has been shown to achieve change in brief interventions: previous studies from our research group [20] have shown significant change in 12 weeks during ACT interventions with clients with psychosis. This is longer than ACT interventions would normally be, but consistent with psychoeducation groups for people with bipolar disorder [6-8]. A future study can address maintenance of effects over time.

\section{Therapists}

We will train and supervise therapists within the service to deliver the intervention. Therapists will be trained to competence in delivery of the manualised intervention, will co-work with a member of the study team, and will be closely supervised by the trial coordinator.

\section{Measures}

\section{Outcome and timeline}

Following informed consent from each participant, all outcomes will be assessed by a trained study researcher. Demographic/clinical characteristics (age, gender, ethnicity, diagnosis) will be assessed at baseline. Outcomes will be assessed at baseline (0 weeks), then at 10 weeks and 14 weeks post randomisation (Table 1 ).

\section{Trial and intervention feasibility parameters}

The feasibility of the trial will be assessed using recruitment, randomisation and attrition rates. Intervention feasibility will be evaluated by treatment adherence and patient satisfaction.

\section{Primary outcome}

The proposed primary outcome will be psychological wellbeing assessed by the Brief Quality of Life in Bipolar Disorder (Brief QoL.BD) [21]. The Brief QoL.BD is well validated and routinely to assess quality of life in people with bipolar disorder [22, 23].

\section{Secondary outcomes}

The proposed secondary outcome of service use will be assessed from the medical records for the 12 months preceding entry to the study and the 3 months during the trial. We will calculate the number of admissions to hospital and contacts with the crisis/home treatment team as well as days under each of these services as an average per month. This is an indication of the severity of the presentation and the cost to the service of care.

\section{Other clinical outcomes}

Mood [24], distress recovery [25, 26] and psychologicalchange processes [27-29] will also be assessed at 0, 10 and 14 weeks. Mood will be assessed sessionally using an un-numbered Likert scale measuring $12 \mathrm{~cm}$, rated from depression/down at one extreme, through normal at $6 \mathrm{~cm}$
Table 1 Balancing ACT: evaluating the effectiveness of psychoeducation and Acceptance and Commitment Therapy (ACT) groups for people with bipolar disorder: Study protocol for a pilot randomised controlled trial. List of measures

Completed at:

\begin{tabular}{|c|c|}
\hline \multicolumn{2}{|l|}{ Service user measures } \\
\hline $\begin{array}{l}\text { Brief Quality of Life in Bipolar Disorder } \\
\text { (Brief QoL.BD: Michalak and Murray, 2009) [21] }\end{array}$ & $1,2,3$ \\
\hline $\begin{array}{l}\text { Clinical Outcomes in Routine Evaluation } \\
\text { Measure (CORE-10; Barkham et al., 2008) [25] }\end{array}$ & $1,2,3$ \\
\hline Valuing Questionnaire (VQ8; Smout et al., 2014) [27] & $1,2,3$ \\
\hline $\begin{array}{l}\text { Acceptance and Action Questionnaire-II } \\
\text { (AAQ-II; Bond et al., 2011) [28] }\end{array}$ & $1,2,3$ \\
\hline $\begin{array}{l}\text { Southampton Mindfulness Questionnaire } \\
\text { (SMQ; Chadwick et al., 2008) [29] }\end{array}$ & $1,2,3$ \\
\hline $\begin{array}{l}\text { Bipolar Recovery Questionnaire } \\
\text { (BRQ; Jones et al., 2013) [26] }\end{array}$ & $1,2,3$ \\
\hline Internal States Scale (ISS; Bauer, 1991) [24] & $1,2,3$ \\
\hline \multicolumn{2}{|l|}{ Researcher-rated measure } \\
\hline $\begin{array}{l}\text { Client Service Receipt Inventory } \\
\text { (CSRl; Beecham and Knapp, 2001) [19] } \\
\text { for the } 12 \text { months preceding and } 3 \text { months } \\
\text { following baseline }\end{array}$ & 1,3 \\
\hline $\begin{array}{l}\text { Service use (average/month) in preceding } \\
12 \text { months and following } 3 \text { months } \\
\text { from baseline }\end{array}$ & \\
\hline \multicolumn{2}{|l|}{ Sessional measures } \\
\hline $\begin{array}{l}\text { Brief Quality of Life in Bipolar Disorder } \\
\text { (Brief QoL.BD: Michalak and Murray, 2009) [21] }\end{array}$ & Sessional \\
\hline
\end{tabular}

Key: Completed at: 1 = baseline ( 0 weeks), 2 = post therapy (10 weeks); 3 = post follow-up (14 weeks)

to manic/high at the other extreme. We also aim to collect qualitative feedback from participants on their experiences of the Balancing ACT group and its impact on their recovery.

\section{Service use and costs}

We will approximate costs by counting average service use/month for the 12 months preceding randomisation [19], and the 3 months following randomisation. Service use variables will include number of psychiatric inpatient admissions, days as an inpatient, number of crisis team contacts, and days under the crisis team.

\section{Sample size}

Resources allow us to recruit 36 participants to our study. Although this number is relatively small it will allow us to gain measures of trial feasibility (such as recruitment rates) and an estimation of the variance following dropout. Sample size estimates for effect size (ES) calculation may start from a total $N$ of 24 [17]. However, ES calculated from small samples are sensitive to inflation [18] and we will take this inflation factor into account when conducting a power analysis for a larger trial. 


\section{Randomisation}

Randomisation will be carried out after consent to participate in the trial has been given and the baseline assessment has been carried out. Participants will be randomised through an independent web-based service provided by UKCRG registered King's Clinical Trials Unit (Reg. No. 053). The randomisation procedure will employ methods to maintain pre-randomisation allocation concealment. We will stratify by site for logistical reasons, so that treatment cases are equitably allocated across sites.

\section{Blinding procedure}

We will not be able to blind participants to treatment group. Similarly, the therapists cannot be blind to allocation as they will deliver the intervention. However, the research workers completing the outcome measurements will be blinded to treatment allocation. Should they be accidentally unblinded during the assessment, we will record which outcomes were completed blind and allocate a new assessor for any subsequent assessments. Post-randomisation assessors will work separately from the research and clinical teams to minimise the likelihood of unblinding. We will report any instances of unblinding in subsequent publications. The end of the trial will be defined as the last follow-up at 14 weeks. The trial statistician will also be blinded to treatment group for the analysis of clinical and functional outcomes.

\section{Data monitoring}

Based on pre-pilot studies we do not anticipate risks to participant safety as a direct result of the study. We will not be conducting any interim data analysis and will not convene a separate Data Monitoring Committee. The trial may be prematurely discontinued by the sponsor, REC or chief investigator (with the agreement of the sponsor and the REC) on the basis of new safety information or for other reasons given by the sponsor or the REC. As the study is a small pilot, we will not convene a Trial Steering Committee. The study will be subject to the standard local and national governance frameworks of the sponsor, the Promoting Recovery Services and research coordination, the clinical research network and the REC.

\section{Data management}

We will use paper assessment packs and enter data into electronic databases which include: (1) baseline demographics, (2) repeated clinical measures, (3) sessional therapy measures and (4) therapy delivery and adherence. Outcome assessments will be carried out by researchers who do not have access to therapy or feedback data. Data will be checked and cleaned against original paper copies and a final database returned to the statistician, who will combine with allocation data for analysis.

Patient data will be pseudonymised for the duration of the study and fully anonymised thereafter. Fully identifiable personal details will be kept on paper in a locked filing cabinet in a locked or occupied office; on secure NHS computers; and encrypted on password-protected computers. All trial data will be stored in line with the Data Protection Act [30].

The allocation database will be accessible only to the study randomiser (who will not conduct post-baseline assessments) and the trial statistician until the study is completed.

\section{Safety monitoring and adverse event reporting}

We do not anticipate safety concerns arising as a direct result of the therapy, which is usually perceived as helpful by service users. However, we will monitor adverse events, defined as any deterioration in psychosocial functioning, irrespective of severity, carefully as one of our outcomes. All adverse events will be reported to the clinical team to ensure adequate care, and will be documented by the study team. Events considered by the participant, clinical service or research team to be unexpected and related to the study will be reviewed for seriousness. Serious adverse events that are related to the trial will be reported to the trial sponsor and the REC within 15 days.

\section{Statistical analysis}

In accordance with the Consolidated Standards of Reporting Trials (CONSORT) principles, we will report all participant flow in the study. The aim of the analysis will be to describe the feasibility and acceptability of the trial ACT/PE intervention and estimate effect sizes for group differences in attitude, adherence, clinical and quality of life outcomes. Binary outcomes will be analysed using logistic regression, chi-square or Fisher's exact test if expected values were small. Continuous outcome scores will be analysed using analysis of covariance (ANCOVA) models in which the mean group difference will be adjusted for baseline outcome scores. Clustering for patients within therapy groups will be accounted a random effect for cluster in the ANCOVA models. Effect sizes will be derived from standardised regression coefficients in which treatment group differences, adjusted for outcomes scores at baseline, will be divided by the standard deviation of the outcomes. Following Cohen's guidelines, effect sizes will be treated as small $(E S=0.2)$, moderate $(E S=0.5)$ or large $(E S=0.8)$.

As a pilot study, the sample size may not give sufficient power to reject null hypotheses; however, we will derive inferential statistics on the intention-to-treat principle. As the sample size is small and regression 


\begin{tabular}{|c|c|c|c|c|c|}
\hline \multicolumn{3}{|l|}{ Balancing ACT } & \multicolumn{3}{|c|}{ STUDY PERIOD } \\
\hline \multirow[b]{2}{*}{ TIME POINT } & \multirow{2}{*}{$\begin{array}{l}\text { Enrolment } \\
\begin{array}{c}\text { Completed } \\
\text { within four } \\
\text { weeks }\end{array}\end{array}$} & \multirow{2}{*}{$\begin{array}{c}\text { Allocation } \\
\text { Within two } \\
\text { weeks of } \\
\text { baseline, } \\
\text { O weeks }\end{array}$} & \multicolumn{2}{|c|}{$\begin{array}{c}\text { Post- } \\
\text { allocation }\end{array}$} & \multirow{2}{*}{\begin{tabular}{|l} 
Close-out \\
26-weeks
\end{tabular}} \\
\hline & & & $\begin{array}{l}0-10 \\
\text { weeks }\end{array}$ & $\begin{array}{l}14 \\
\text { weeks }\end{array}$ & \\
\hline $\begin{array}{r}\text { ENROLMENT: Routine eligibility } \\
\text { screen }\end{array}$ & $\mathrm{x}$ & & & & \\
\hline Informed consent/assent & $\mathrm{X}$ & & & & \\
\hline Allocation & & $\mathrm{X}$ & & & \\
\hline $\begin{array}{r}\text { INTERVENTION: } \\
A C T / P E+T A U\end{array}$ & & & $\mathrm{x}$ & & \\
\hline Treatment as usual (TAU) & & & & $\mathrm{X}$ & \\
\hline $\begin{array}{r}\text { ASSESSMENTS: Primary Outcome: } \\
\text { Brief } Q o L .{ }^{1}\end{array}$ & $\mathrm{x}$ & & $\mathrm{x}$ & $\mathrm{X}$ & \\
\hline $\begin{array}{r}\text { Secondary Outcomes: } \\
\text { Mood }^{2}, \text { distress recovery }\end{array}$ & $\mathrm{x}$ & & $\mathrm{x}$ & $\mathrm{x}$ & \\
\hline $\begin{array}{l}\text { Other Secondary Outcomes: } \\
\text { Psychological change process }{ }^{4}\end{array}$ & $\mathrm{x}$ & & & & \\
\hline $\begin{array}{r}\text { Other Secondary Outcomes } \\
\text { Service use }\end{array}$ & $\begin{array}{l}\mathrm{X} \text { (12 months } \\
\text { preceding } \\
\text { baseline) }\end{array}$ & & $\mathrm{x}$ & & $\begin{array}{c}\mathrm{X}(3 \\
\text { months } \\
\text { following } \\
\text { baseline) }\end{array}$ \\
\hline
\end{tabular}

Fig. 2 Balancing ACT: evaluating the effectiveness of psychoeducation and Acceptance and Commitment Therapy (ACT) groups for people with bipolar disorder: study protocol for a pilot randomised controlled trial. Schedule of enrolment, interventions and assessments

assumptions may not be met and we will use robust methods, such as bootstrap resampling, to derive standard errors and bias-corrected accelerated confidence intervals. Missing data will be described per outcome and if possible mechanisms driving missingness accounted for in the statistical model under the assumption that data is missing at random and with sensitivity analyses. All statistical tests will be two-sided and confidence interval level set at 95\%. STATA 14.1 (StataCorp, College Station, TX, USA) will be used to analyse the data.

\section{Discussion}

We have adhered to Standard Protocol Items: Recommendations for Interventional Trials (SPIRIT) [31, 32] guidance in devising and reporting our protocol (Fig. 2; SPIRIT Checklist with information sheets and consent forms for participants are included as an Additional file 1). The trial is funded until the end of July 2018. The study will be the first study of its kind examining ACT and psychoeducation groups for people with bipolar disorder and/or symptoms. Should the intervention prove feasible, acceptable and potentially helpful cost-effective addition to routine care, we will apply for funding for a larger-scale evaluation. If outcomes suggest that the intervention does not show promise in terms of potential clinical improvement, we will continue to refine the intervention, based on participant feedback, further consultation, and our process measures, before attempting a further pilot. Findings will be limited by the pilot nature of the study, including the small sample size, location in a single service and opportunistic recruitment strategy. The therapy will be compared to treatment as usual and not to an alternative psychological intervention, so any effects found may be non-specific. We are not assessing longevity of effects in this study: further research will be required to ascertain longer-term outcomes.

We plan to disseminate our findings via local academic and clinical networks, through conference presentation and publication. Authorship will be restricted to those making a substantial contribution to the specific publication.

\section{Status}

Participants began to enter the trial in April 2017. The first participant was randomised on 10 May 2017. Recruitment will continue until end of October 2017. We anticipate that final primary outcome data will be collected by the end of February 2018.

\section{Additional file}

Additional file 1: Standard Protocol Items: Recommendations for Interventional Trials (SPIRIT) 2013 Checklist: recommended items to address in a clinical trial protocol and related documents*. (DOC $121 \mathrm{~kb}$ )

\section{Acknowledgements}

This paper summarises independent research funded by Guys and St Thomas' Charity (Reference EFT151106). The views expressed are those of the authors and not necessarily those of the NHS. The study is sponsored by the South London and Maudsley NHS Foundation Trust. The study design, collection, management, analysis and interpretation of data, writing of the report; and the decision to submit the report for publication, are all the responsibility of the study team, not the funders or sponsors, and will remain so.

\section{Declarations}

The study has been reviewed and given a favourable opinion by the London and Surrey Borders National Research Ethics Committee (reference 17/LO/0445). 
No personal data is reported, so no consent for publication is required. The datasets used and/or analysed during the current study are available from the corresponding author on a reasonable request.

\section{Authors' contributions}

EOD is the principle investigator for the study. JH is the trial statistician. SJ and MR are the lead clinicians and consultant psychologists in the Promoting Recovery Psychology Services, advising on the acceptability of the study to teams. DOK is the study research worker and is responsible for the recruitment of participants and data management. AC, SN and LB are experts in the development and dissemination of psychological therapies for adults with bipolar disorder and will be facilitating the ACT/PE groups. EM is an expert in using Acceptance and Commitment Therapy and FK is an expert in psychological interventions for people with bipolar disorder. Both have provided advice and guidance on the development of the treatment manual. All authors contributed to the design of the study protocol and were involved in writing the manuscript. All authors read and approved the final manuscript.

\section{Competing interests}

The authors declare that they have no competing interests.

\section{Publisher's Note}

Springer Nature remains neutral with regard to jurisdictional claims in published maps and institutional affiliations.

\begin{abstract}
Author details
'South London and Maudsley NHS Foundation Trust, London SE5 8AZ, UK. 2Department of Biostatistics, King's College London, Institute of Psychiatry, Psychology and Neuroscience, London SE5 8LY, UK. ' ${ }^{3}$ LaTrobe University, Melbourne, Australia. ${ }^{4}$ Department of Psychology, King's College London, Institute of Psychiatry, Psychology and Neuroscience, London SE5 8AF, UK. ${ }^{5}$ South London and Maudsley NHS Foundation Trust, 308-312 Brixton Road, London SW9 6AA, UK.
\end{abstract}

Received: 29 September 2017 Accepted: 5 July 2018 Published online: 13 August 2018

\section{References}

1. Mansell W, Morrison AP, Reid G, Lowens I, Tai S. The interpretation of, and responses to, changes in internal states: an integrative cognitive model of mood swings and bipolar disorders. Behav Cogn Psychother. 2007;35(05): 515-39.

2. NICE Bipolar Disorder: Assessment and Management. NICE clinical guideline CG185. 2014. Available at www.nice.org.uk/CG185 [NICE guideline].

3. Mansell W, Tai S, Clark A, Akgonul S, Dunn G, Davies L, et al. A novel cognitive behaviour therapy for bipolar disorders (Think Effectively About Mood Swings or TEAMS): study protocol for a randomized controlled trial. Trials. 2014;15(1):405.

4. Treuer T, Tohen M. Predicting the course and outcome of bipolar disorder: a review. Eur Psychiatry. 2010;25(6):328-33.

5. Gitlin MJ, Swendsen J, Heller TL, Hammen C. Relapse and impairment in bipolar disorder. Am J Psychiatry. 1995;152(11):1635.

6. Scott J, Colom F, Vieta E. A meta-analysis of relapse rates with adjunctive psychological therapies compared to usual psychiatric treatment for bipolar disorders. Int J Neuropsychopharmacol. 2007;10(01):123-9.

7. Smith DJ, Griffiths E, Poole R, Di Florio A, Barnes E, Kelly MJ, et al. Beating bipolar: exploratory trial of a novel Internet-based psychoeducational treatment for bipolar disorder. Bipolar Disord. 2011;13(5-6):71-577.

8. Miklowitz DJ. Adjunctive psychotherapy for bipolar disorder: state of the evidence. Am J Psychiatr. 2008;165(11):1408-19.

9. NICE. Schizophrenia: core interventions in the treatment and management of schizophrenia in adults in primary and secondary care, NICE clinical guideline; 2009. p. 82.

10. Hayes SC, Strosahl K, Wilson KG. Acceptance and commitment therapy: an experiential approach to behavior change. New York: Guilford Press; 1999.

11. Öst LG. The efficacy of acceptance and commitment therapy: an updated systematic review and meta-analysis. Behav Res Ther. 2014;61:105-21.

12. Davis ML, Morina N, Powers MB, Smits JAJ, Emmelkamp PMG. A metaanalysis of the efficacy of acceptance and commitment therapy for clinically relevant mental and physical health problems. Psychother Psychosom. 2015; 84(1):30-6.
13. Bach $P$, Hayes SC. The use of acceptance and commitment therapy to prevent the rehospitalization of psychotic patients: a randomized controlled trial. J Consult Clin Psychol. 2002;70(5):1129.

14. Pankowski S, Adler M, Andersson G, Lindefors N, Svanborg C. Group acceptance and commitment therapy (ACT) for bipolar disorder and COexisting anxiety_an open pilot study. Cogn Behav Ther. 2017:46(2):114-28.

15. Gergel T, Owen GS. Fluctuating capacity and advance decision-making in bipolar affective disorder-self-binding directives and self-determination. Int J Law Psychiatry. 2015;40:92-101.

16. Kurtz MM, Gerraty RT. A meta-analytic investigation of neurocognitive deficits in bipolar illness: profile and effects of clinical state. Neuropsychology. 2009;23(5):551.

17. Julious SA. Sample size of 12 per group rule of thumb for a pilot study. Pharm Stat. 2005;4(4):287-91.

18. Sim J, Lewis M. The size of a pilot study for a clinical trial should be calculated in relation to considerations of precision and efficiency. J Clin Epidemiol. 2012;65(3):301-8.

19. Beecham J, Knapp M. Costing psychiatric interventions. In: Measuring mental health needs. Thornicroft G, editor. London: Gaskell; 2001. p. 200-224.46.

20. Johns LC, Oliver JE, Khondoker M, Byrne M, Jolley S, Wykes T, et al. The feasibility and acceptability of a brief Acceptance and Commitment Therapy $(A C T)$ group intervention for people with psychosis: the 'ACT for life' study. J Behav Ther Exp Psychiatry. 2016;50:257-63.

21. Michalak EE, Murray G. Development of the QoL. BD: a disorder-specific scale to assess quality of life in bipolar disorder. Bipolar Disord. 2010;12(7):727-40.

22. Jones SH, Smith G, Mulligan LD, Lobban F, Law H, Dunn G, et al. Recoveryfocused cognitive-behavioural therapy for recent-onset bipolar disorder: randomised controlled pilot trial. Br J Psychiatry. 2015;206(1):58-66.

23. Xiao L, Gao Y, Zhang L, Chen P, Sun X, Tang S. Validity and reliability of the Brief version of Quality of Life in Bipolar Disorder (Brief QoL. BD) among Chinese bipolar patients. J Affect Disord. 2016;193:66-72.

24. Bauer M, Crits-Christoph P, Ball W, Dewees E, McAllister T, Alahi P, et al. Independent assessment of manic and depressive symptoms by self-rating: scale characteristics and implications for the study of mania. Arch Gen Psychiatry. 1991;48:807-12.

25. Barkham M, Bewick B, Mullin T, Gilbody S, Connell J, Cahill J, et al. The CORE-10: a short measure of psychological distress for routine use in the psychological therapies. Counsell Psychother Res J. 2013;13:3-13.

26. Jones S, Mulligan LD, Higginson S, Dunn G, Morrison AP. The Bipolar Recovery Questionnaire: psychometric properties of a quantitative measure of recovery experiences in bipolar disorder. J Affect Disord. 2013;147(1):34-43.

27. Smout M, Davies M, Burns N, Christie A. Development of the Valuing Questionnaire (VQ). J Contextual Behav Sci. 2014;3(3):164-72.

28. Bond FW, Hayes SC, Baer RA, Carpenter KM, Guenole N, Orcutt HK, et al. Preliminary psychometric properties of the Acceptance and Action Questionnaire-II: A revised measure of psychological inflexibility and experiential avoidance. Behav Ther. 2011;42(4):676-88.

29. Chadwick P, Hember M, Symes J, Peters E, Kuipers E, Dagnan D. Responding mindfully to unpleasant thoughts and images: reliability and validity of the Southampton Mindfulness Questionnaire (SMQ). Br J Clin Psychol. 2008; 47(4):451-5.

30. The Data Protection Act. 1998. http://www.legislation.gov.uk/ukpga/1998/ 29/contents. Accessed 8 Jan 2017.

31. Chan A-W, Tetzlaff JM, Altman DG, Laupacis A, Gøtzsche PC, Krleža-Jerić K, Hróbjartsson A, Mann H, Dickersin K, Berlin J, Doré C, Parulekar W Summerskill W, Groves T, Schulz K, Sox H, Rockhold FW, Rennie D, Moher D. SPIRIT 2013 Statement: defining standard protocol items for clinical trials. Ann Intern Med. 2013;158:200-7.

32. Chan A-W, Tetzlaff JM, Gøtzsche PC, Altman DG, Mann H, Berlin J, Dickersin K, Hróbjartsson A, Schulz KF, Parulekar WR, Krleža-Jerić K, Laupacis A, Moher D. SPIRIT 2013 explanation and elaboration: guidance for protocols of clinical trials. BMJ. 2013;346:e7586. 